\title{
THE USE OF MAGNETIC RESONANCE IN THE DIVISION OF THE CORPUS CALLOSUM IN ADULT PATIENTS WITH SYSTEMIC LUPUS ERYTHEMATOSUS ACCORDING TO THE AGE OF ONSET OF THE DISEASE
}

Paulo Rogério Julio ${ }^{1}$, Carla Helena Cappello ${ }^{1}$, Simone Thiemi Kishimoto ${ }^{1}$, Renan Bazuco Frittoli ${ }^{1}$, Roberto Marini ${ }^{1}$, Fernando Cendes $^{1}$, Lilian Tereza Lavras Costallat ${ }^{1}$, Leticia Rittner ${ }^{1}$, Simone Appenzeller ${ }^{1, *}$

1.Universidade Estadual de Campinas, Campinas (SP), Brazil.

*Corresponding author: appenzellersimone@gmail.com

\section{BACKGROUND}

Corpus callosum (CC) atrophy is frequently observed in childhood-onset systemic lupus erythematosus (CSLE) and adult-onset (aSLE). Disease duration, corticosteroid use and neuropsychiatric manifestations have been identified as risk factors for CC atrophy. Since CC receives fibers from different brain areas, the aim of this study was to evaluate which area of the CC was more frequently affected in SLE according to age of disease-onset.

\section{MATERIALS AND METHOD}

We selected 71 patients with cSLE [mean age 24.7 years (SD 4.6) and disease duration 11.8 years (SD 4.8)] and 49 patients with SLE onset in adulthood [mean age of 33.2 (SD 3.7)] and disease duration 11.3 years (SD 4.05)], and 58 healthy controls (HC) [mean age 29.9 years (SD 4.1)]. All individuals underwent three-tesla magnetic resonance imaging (3T MRI) and mid-sagittal axial slice was used for automatic segmentation of the $\mathrm{CC}$ in five parts. All patients underwent a neurological and psychiatric evaluation.

\section{RESULTS}

Both aSLE and CSLE had similar CC area on T1, whereas decreased FA $(p<0.01)$ and increased values of $M D(p<0.01)$ were observed in the $C C$ and in the parcels $B, C, D, E$, all with $p<0.01$ when compared to $H C$. We observed reduced $C C$ area in cSLE in the areas $B(p<0.001), C(p<0.001), D(p<0.001), E(p<0.001)$. We found an association between NP (neuropsychiatry) manifestations with decreased values of FA and increased values of $M D$ in the group of patients with cSLE when compared with the group of aSLE.

\section{CONCLUSION}

Although a similar area was observed in cSLE when compared to aSLE, we observed significant microstructural damage in the white matter, especially in the most posterior segments of the CC. These findings suggest that parietal, temporal and occipital area are more frequently involved in cSLE when compared to aSLE. 